\title{
Test of Anderson-Stuart Model and the "Universal" Conductivity in Rubidium and Cesium Silicate Glasses
}

\author{
Marcio Luis Ferreira Nascimento, \\ Department of Materials Engineering, Federal University of São Carlos, 13565-905, São Carlos-SP, Brazil
}

Eduardo do Nascimento and Shigueo Watanabe

Institute of Physics, University of São Paulo, 05508-900 São Paulo-SP, Brazil

Received on 2 February, 2005

\begin{abstract}
An analysis and brief discussion of experimental ionic conductivity $\sigma$ and activation energy $E_{A}$ in the binary rubidium and cesium silicate systems is presented, exemplified on 23 and 30 glasses respectively, in a wide composition range (5-45 $\mathrm{Rb}_{2} \mathrm{O}$ and $\mathrm{Cs}_{2} \mathrm{O}$ mole\%). The Anderson and Stuart model has been considered to describe the variation of activation energy $E_{A}$ with alkali concentration in both alkali-silica systems. In this analysis were considered experimental parameters, like shear modulus $G$ and relative dielectric permittivity $\varepsilon$. An "universal" finding is obtained using $\log \sigma \times E_{A} / k_{B} T$ in 51 of 53 glasses considering both alkali systems, where $E_{A}$ is the activation energy for conduction, $k_{B}$ is the Boltzmann constant and $T$ is the absolute temperature. This strong correlation by more than 13 (Rb-based glasses) and 15 (Cs-based glasses) orders of magnitude means that $\sigma$ is governed mainly by $E_{A}$. An explanation for this behavior links ionic conductivity and microscopic structure.
\end{abstract}

\section{INTRODUCTION}

Since the discovery of fast ionic glass conductors there has been a large interest in applications, as new solid-state batteries, sensors, "smart windows", and the search for an "universal" theory of ion-transport in glassy materials. Despite considerable experimental and theoretical attempt, there is currently no consensus regarding the diffusion mechanism [1]. In order to understand the diffusion transport it is essential to find a connection between the microscopic structure and the ionic conductivity. Thus, several transport models have been proposed, and they vary from thermodynamics with principles in models for liquid electrolytes, such as the weak electrolyte model [2], to models based on solid state concepts such as the jump diffusion model [3], the strong electrolyte (AndersonStuart) model [4], and the dynamic structure model [5].

Most of these models involve specific or indirect assumptions about the microscopic structure in general and the distribution or local environment of the mobile cations in particular. It is well know that the ionic conductivity increases rapidly when a network glass former is modified by the addition of a metal oxide, resulting in a lowering of the activation energy for ionic conduction.

Among the main models, the Anderson \& Stuart (A-S) [4] is considered to be the most directly related to physically meaning parameters, such as ionic radii, relative dielectric permittivity and the elastic modulus. But at the time of the A-S theory no much experimental data were available. For the rubidium and cesium silicate systems there is no analysis performed considering so wide composition range.

The present paper reports on the ionic conductivities and activation energies of glasses in $\mathrm{Rb}_{2} \mathrm{O}-\mathrm{SiO}_{2}$ and $\mathrm{Cs}_{2} \mathrm{O}-\mathrm{SiO}_{2}$ systems, with the purpose to correlate new proposals to activation energy with composition using experimental parameters data, like shear modulus $G$ and relative dielectric permittivity $\varepsilon$. These glasses correspond to most of all available conductivity data measured up to now. Also, an "universal" finding is obtained in this binary system when one uses $\log \sigma \times E_{A} / k_{B} T$.

\section{RESULTS AND DISCUSSION}

Ionic conductivity $\sigma$ in glass is a thermally activated process of mobile ions that overcome a potential barrier $E_{A}$, of the form:

$$
\log \sigma=\log \sigma_{0}-(\log e) E_{A} / k_{B} T
$$

where $\sigma_{0}$ is the pre exponential factor. For most glasses, conduction is solely due to movement of a single ionic species, anionic or cationic. The main factors that are believed to control the $\sigma$ magnitude in glasses are associated with the biding energies holding the mobile ions in their equilibrium (metastable) sites and the migration energy barriers that the ions face because of the volume requirements for their movement. In fact, it is supposed that the activation energy barrier $E_{A}$ represents the binding energy that holds the ions in their equilibrium (metastable) sites, and once an ion has dissociated away from its site it is thought to experience no other energy barrier(s) to motion.

As will be presented below, there is some disparity between experimental data on similar glasses from different authors. At first sight, the variation in values of $\sigma$ and activation energies implies in different experimental procedures, but is also possible to obtain the same variations from chemical and/or structural states of the glass samples. Obviously, the detailed microscopic structure should be different for different kinds of ion conducting glasses at same temperatures and same alkali concentration. 


\section{A. The Anderson-Stuart Model}

Anderson \& Stuart [4] have provided a quantitative analysis of the conduction energetics in an ion-conducting glass. In this model the activation energy for conduction may be divided in two parts: the electrostatic binding energy of the original site $E_{b}$, and the strain energy, $E_{s}$, required to move an ion from one site to another. $E_{b}$ represents the necessary energy to remove a cation from a non-bridging oxygen site, and $E_{S}$ describes the dilate of the structure as the ion moves from one site to another. The basic idea is that an ion (in this case $\mathrm{Rb}^{+}$ or $\mathrm{Cs}^{+}$) makes a simple jump from one site to another, and passes through a "doorway" which opens as it passes through, where cations sites require only the presence of non-bridging oxygens (Eq. (2)).

$$
E_{A}=\frac{\beta z z_{0} e^{2}}{\gamma\left(r+r_{O}\right)}+4 \pi G r_{D}\left(r-r_{D}\right)^{2}
$$

where $z$ and $z_{0}$ are the valence of the mobile ion and the fixed counterion in this case alkali and oxygen, respectively; $r$ and $r_{O}$ are the corresponding Pauling ionic radii for $\mathrm{Rb}^{+}$or $\mathrm{Cs}^{+}$ and $\mathrm{O}^{2-}, e$ is the electronic charge and $r_{D}$ is the effective radius of the (unopened) doorway.

The parameters of interest in the A-S model are the elastic modulus $(G)$, a "Madelung" constant $(\beta)$, which depends on how far apart the ions are, and a covalency parameter $(\gamma)$, which indicates the degree of charge neutralization between the ion and its immediate neighbors. In their original paper [4], Anderson \& Stuart assumed that this covalency parameter can be taken equal to the relative dielectric permittivity (ع). McElfresh \& Howitt [7] have reexamined the $E_{s}$ term, and have suggested a modified form that overcomes certain limitations of the original A-S theory. In summary, McElfresh $\&$ Howitt [7] include the jumping distance $\lambda$ as a better parameter (Eq. (3)).

$$
E_{A}=\frac{\beta z z_{0} e^{2}}{\gamma\left(r+r_{O}\right)}+4 \pi G \lambda\left(r-r_{D}\right)^{2}
$$

Following A-S theory, the experimental $G$ and $\varepsilon$ parameters are presented in Figs. $1 a-b$ and Figs. $2 a-b$, respectively for both alkali systems. At least, we consider $\beta$ as it was done by Anderson \& Stuart [4]:

$$
\beta=\frac{a-r}{b}
$$

where $r$ is a value given in $\ddot{a n g s t r o m}$, also with $a$ and $b$, that will be defined below.

In this work the authors considered two assumptions: $(i)$ $r_{D}$ fitting all data, as suggested by A-S theory. (ii) $\lambda$ fitting all data, following McElfresh \& Howitt's suggestion [7], Eq. (3). In the second case one should also consider $r_{D}$ as a fitting parameter, just for comparison.

From Figs. $1 a-b$, the shear modulus $G$ from Nemilov [8] and Shelby \& Day [10] showed a decrease with increasing rubidium composition. Data from Nemilov [8], Takahashi \&
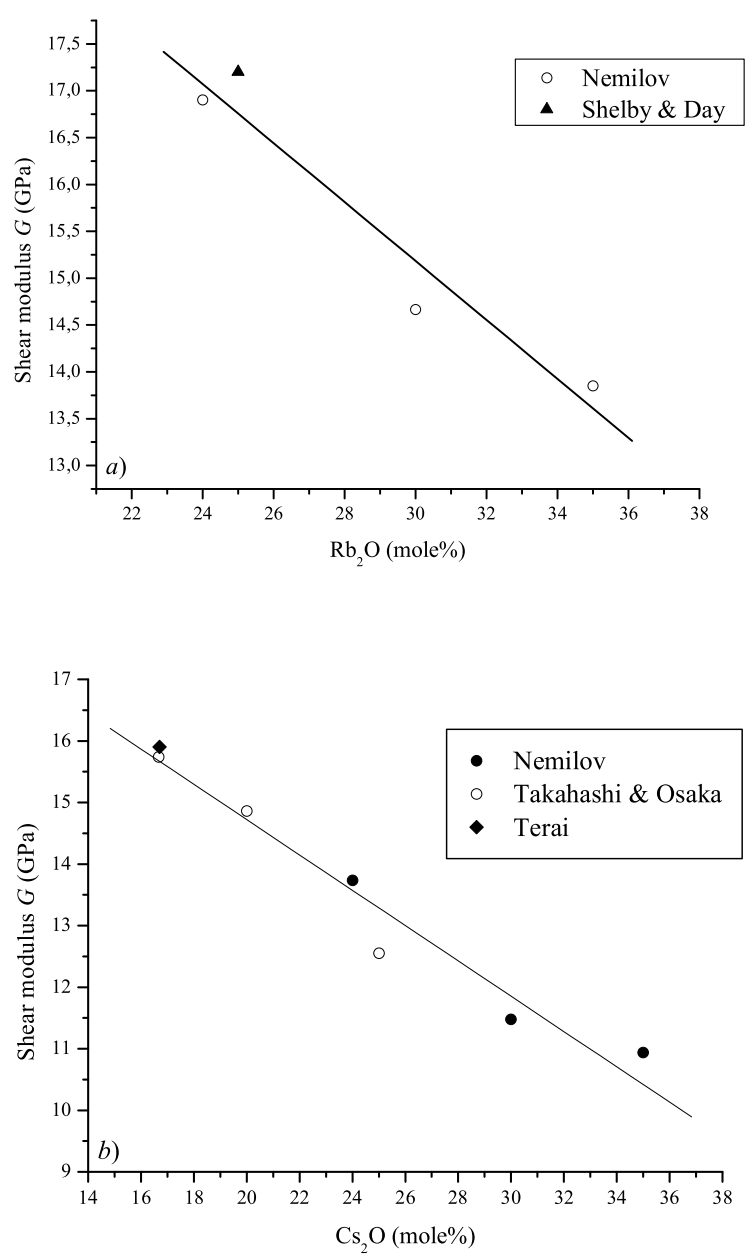

FIG. 1: Measured shear modulus $(G$, in $\mathrm{GPa})$ in $\mathrm{Rb}_{2} \mathrm{O}-\mathrm{SiO}_{2}(a)$ and $\mathrm{Cs}_{2} \mathrm{O}-\mathrm{SiO}_{2}(b)$ systems $[8,10,11,15]$.

Osaka [11] and Terai [15] presented same decreasing behavior with increasing cesium composition. In this work we proposed that $G$ follow a linear fit (Eq. (5)):

$$
G=G_{0}-\frac{d G}{d n} n
$$

where $G_{0}=(24.6 \pm 1.7) \mathrm{GPa}, n$ is the $\mathrm{Rb}_{2} \mathrm{O}$ mole\% composition and $d G / d n=(0.315 \pm 0.060) \mathrm{GPa} / \mathrm{mole} \%$, with correlation factor $R^{2}=0.96$. For cesium silicate system, we found $G_{0}=(20.46 \pm 0.69) \mathrm{GPa}, n$ is the $\mathrm{Cs}_{2} \mathrm{O}$ mole\% composition and $d G / d n=(0.287 \pm 0.027) \mathrm{GPa} / \mathrm{mole} \%$, with correlation factor $R^{2}=0.98$

From Figs. $2 a-b$, the relative dielectric permittivity $\varepsilon$ from Amrhein [12] and Charles [16] showed a small and monotonic increase with increasing rubidium composition; but Charles's [16] and Matusita et al.'s [15] data do not follow the linear increasing as measured by Amrhein [12] and Hakim \& Uhlmann [13] in cesium composition. All linear fits follow Eq. (6): 

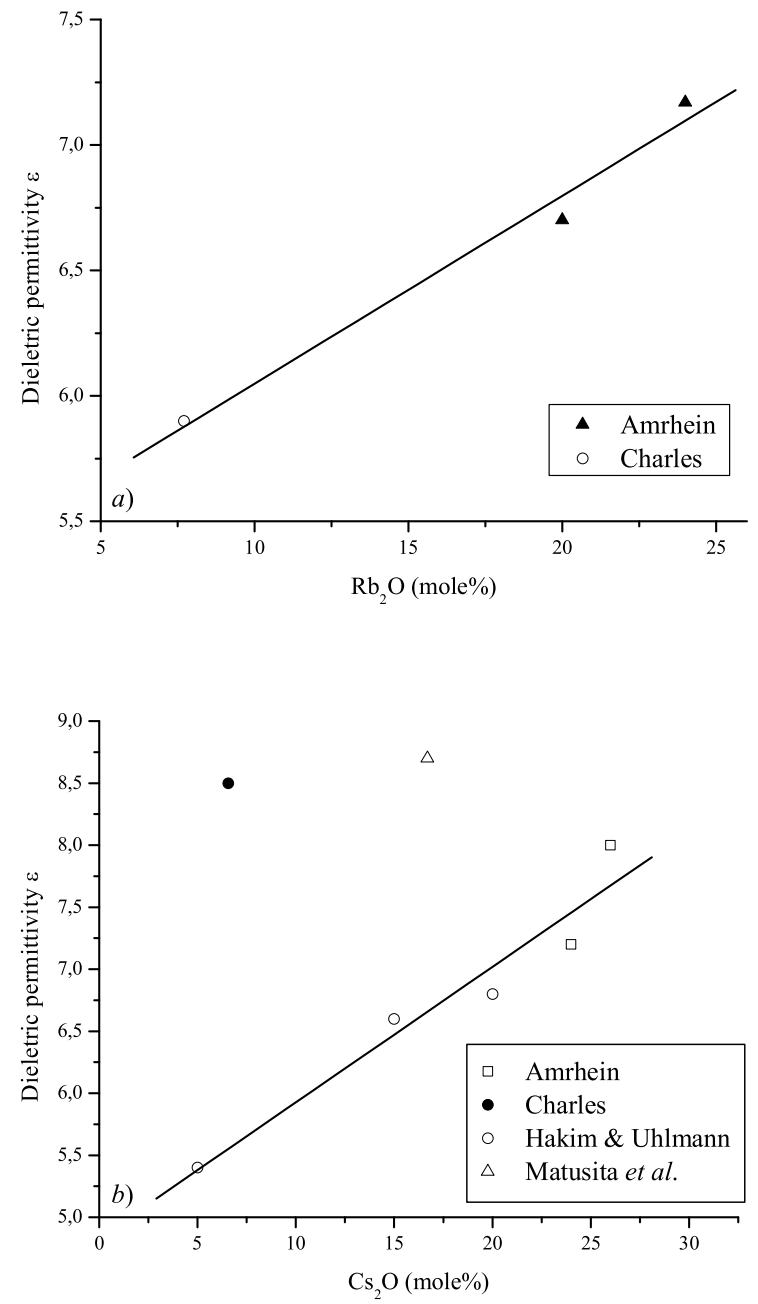

FIG. 2: Experimental relative dielectric permittivity values $(\varepsilon)$ in $\mathrm{Rb}_{2} \mathrm{O}-\mathrm{SiO}_{2}(a)$ and $\mathrm{Cs}_{2} \mathrm{O}-\mathrm{SiO}_{2}(b)$ systems [12-16]. Data from Charles [16] and Matusita et al. [14] in (b) were discarded from analysis.

$$
\varepsilon=\varepsilon_{0}+\frac{d \varepsilon}{d n} n
$$

where $R^{2}=0.99, \varepsilon_{0}=(5.30 \pm 0.19)$ and $d \varepsilon / d n=(0.075 \pm$ $0.010) /$ mole $\%$ considering rubidium system, and $\varepsilon_{0}=$ $(4.83 \pm 0.33)$ and $d \varepsilon / d n=(0.109 \pm 0.017) /$ mole $\%$, with $R^{2}=0.97$ in the cesium system. It is recognized that these assumptions of a similitude of form presented in Eqs. (5-6) may provide inadequate descriptions of changes in $G$ and $\varepsilon$ with alkali concentration, but it seems to represent only an approximation.

The variation of activation energy $E_{A}$ with alkali composition over 53 different glasses are shown in Figs. $3 a-b$. A careful analysis was done in all data to find some possible discrepancies about scattering. For example, the activation energies $E_{A}$ in both systems which have been measured by Negodaev et al. [24] differ considerably from others.

At high alkali content, the samples are hygroscopic, and special precautions in the preparation and measuring procedures must be taken to ensure the absence of surface or bulk proton conduction (from water content). These processes would lead to higher $\sigma$ conductivities, and consequently to lower $E_{A}$ activation energies. While the origin of the scatter in the various reported values of the activation energy $E_{A}$ cannot now be identified, a comparison of the different data sets provides a feeling for the accuracy without detailed specification of their chemical and structural states.

Besides appreciable scatter, effects of glass composition on $E_{A}$ could be parametrized by A-S theory. Even, this model could be interesting to apply in alkali silicate glasses to preview, for example, the amount of $E_{A}$ with alkali composition (Figs. $3 a-b$ ). The A-S model calculations of $E_{A}$ gives a better agreement at medium range alkali composition and the departure is more at lower and higher alkali composition. In fact, the scattering values in $E_{A}$ should correspond to chemical and/or thermal history, more than by measurement procedure. Indeed, Figs. $3 a-b$ are dealing with with different structures considering a fixed alkali composition, as shown below.

About the fitting, in the first case were fixed radii values ( $r=1.48 \AA$ And $r=1.69$ Åfor rubidium and cesium ions, respectively, with $r_{O}=1.4 \AA$, see Figs. $3 a-b$, full line). The fitting parameter was the "doorway" radius, that resulted in $r_{D}=1 \AA$ And $r_{D}=1.12 \AA$, respectively. The second assumption showed the "doorway" radius value giving $r_{D}=1.24$ Åand $r_{D}=1.4$ Åwith the jumping distances $\lambda=4$ Åand $\lambda=4.3 \AA$, respectively, using both $r_{D}$ and $\lambda$ as fitting parameters. On both assumptions were used experimental shear modulus $G$ and the relative dielectric permittivity $\varepsilon$ as described above (Figs. 1-2). The $\beta$ parameter used resulted both in $\beta=0.32$ considering assumptions $i$ and $i i(a=2.59 ; b=3.5$ to rubidium case, and $a=2.24 ; b=1.73$ to cesium case) respectively.

The adjustment for activation energy $E_{A}$ in Figs. $3 a-b$ was performed using a Levenberg-Marquardt non-linear fitting. It is surprising that only one simple theory could fit a lot of data from several authors with different glass preparation processes in a wide range of compositions. Significantly, $E_{b}$ decreases substantially with increasing alkali oxide in both results of Figs. $3 a-b$. One reason concerns with the relative dielectric permittivity $\varepsilon$, that increases with increasing alkali oxide. Figs. $3 a-b$ also shown that $E_{b}$ is higher than $E_{s}$ considering Anderson-Stuart [4] assumption (i) in wide composition range. The McElfresh \& Howitth [7] hypothesis (ii) presented similar results concerning $E_{A}$ as well. It is important to note that the A-S model is limited to only one site energy distribution and with fixed $r_{D}$ to all composition range. However, the model reasonably agrees with with experimental data, describing $E_{A}$ decreasing tendency with alkali oxide composition. 

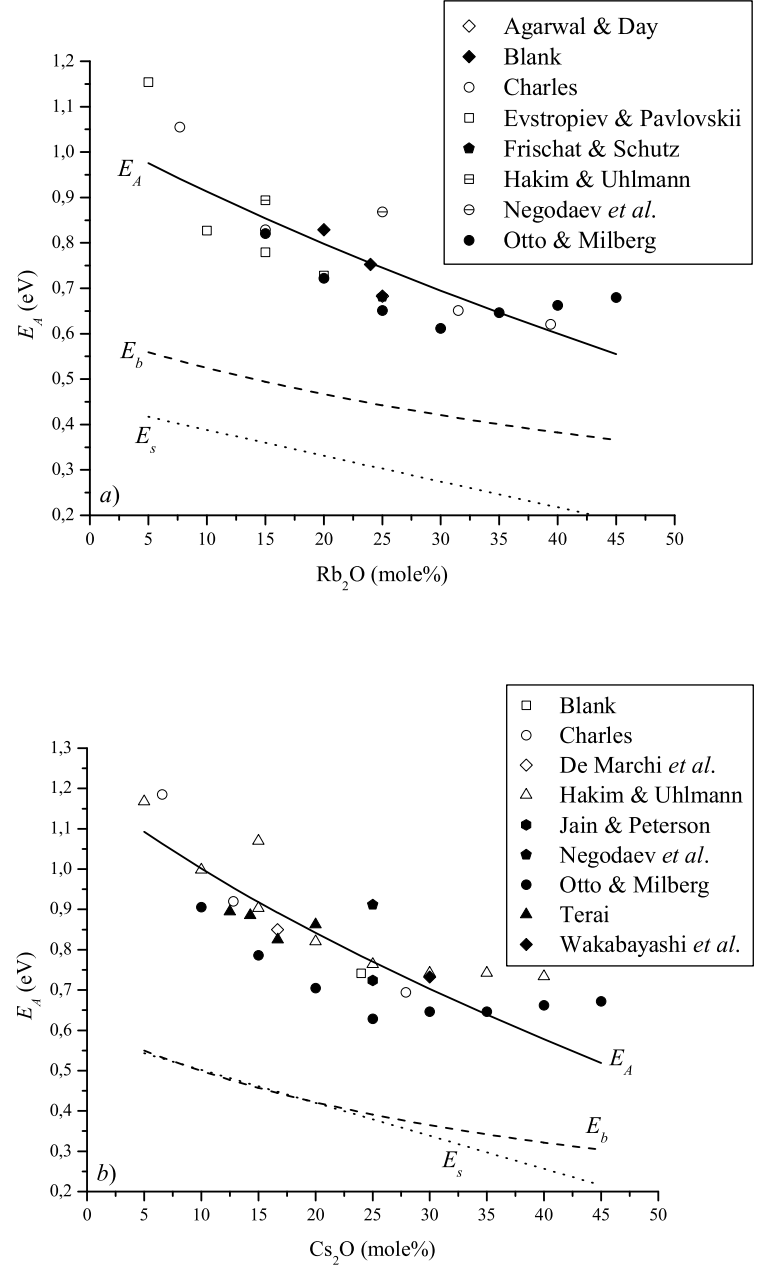

FIG. 3: Non-linear fit (full line) on activation energies $E_{A}$ from various authors of $\mathrm{Rb}_{2} \mathrm{O}-\mathrm{SiO}_{2}(a)$ and $\mathrm{Cs}_{2} \mathrm{O}-\mathrm{SiO}_{2}(b)$ systems considering assumption $(i)[6,15-27] . E_{b}$ is the binding energy (dashed line), and $E_{S}$ is the strain energy (dotted line).

\section{B. The "Universal" Conductivity}

Extensive studies have recently been made for obtaining an "universal" equation from the standpoint of glass structure. For example, Doi [28] presented 17 different glasses (not mentioned) that follows an "universal" conductivity rule. Swenson \& Börjesson [29] proposed a common cubic scaling relation of $\sigma$ with the expansion volumes of the network forming units in salt-doped and -undoped glasses. This fact suggested that the glass network expansion, which is related to the available free volume, is a key parameter determining the increase of the high ionic conductivity in some types of fast ion conducting glasses.

According to Adams \& Swenson [30], the ion conduction should be determined by the ionic motion within an infinite pathway cluster. For various silver ion conducting glasses [3132 ], it was found that the cubic root of the volume fraction $F$ of infinite pathways for a fixed valence mismatch threshold is closely related to both the absolute conductivity and the activation energy of the conduction process:

$$
\log \sigma T \propto \sqrt[3]{F}=\log \sigma_{0}^{\prime}-(\log e) E_{A} / k_{B} T
$$

where $\sigma_{0}^{\prime}$ is the pre exponential factor (in $\mathrm{K} \cdot \mathrm{S} / \mathrm{cm}$ ).

More recently, Nascimento \& Watanabe [33] verified this modified Arrhenius "universal" finding in binary borate glasses, considering both Eqs. (1) and (7). This paper aims to present new results considering just rubidium and cesium binary silicate glasses.

Figs. $4 a-b$ shows modified Arrhenius plots of $\sigma$ for 23 rubidium and 30 for cesium silicate glasses, from $x=5$ to $45 \mathrm{~mol} \%$ in both systems), ranging from $7 \times 10^{-2} \mathrm{~S} / \mathrm{cm}$ to $2 \times 10^{-14} \mathrm{~S} / \mathrm{cm}$ in rubidium and $13 \mathrm{~S} / \mathrm{cm}$ to $3 \times 10^{-16} \mathrm{~S} / \mathrm{cm}$ in cesium systems, all between $20^{\circ} \mathrm{C}$ to $450^{\circ} \mathrm{C}$. The range of activation energy $E_{A}$ lies between 0.61 and $1.15 \mathrm{eV}$ (rubidium) and 0.62 and $1.18 \mathrm{eV}$ (cesium) in all glasses studied, as indicated in Figs. $3 a-b$. These data were compared with the "universal" equation for $\sigma_{0}=50 \mathrm{~S} / \mathrm{cm}$ in Eq. (1). This "universal" equation, following Doy 's sense, appears in Figs. $4 a-b$ as a dashed line, and the dotted lines are the higher and lower limits within one magnitude order.

It is important to note that the same data from Negodaev et al. [24] that diverges from other data in Figs. $3 a-b$ also diverges in Figs. $4 a-b$. In fact, considering so many different binary alkali silicate glasses according to Figs. $4 a-b$ is remarkable so strong correlation between $\sigma$ with $E_{A} / k_{B} T$. It is interesting to note that the increase in ionic conductivity with alkali content is almost entirely due to the fact that the activation energy $E_{A}$ required for a cation jump decreases, as presented in Figs. $3 a-b$. Thus, the term $\sigma_{0}$ in Eq. (1) is largely unaffected upon alkali content.

The fact is that $\sigma$ lies on a single "universal" curve in various alkali silicate glasses, whose conductivities differ by more than 13 orders of magnitude in rubidium system and 15 orders of magnitude in cesium system and in $93 \%$ of the glass systems considered (within just one order of magnitude). Therefore, if one measure $\sigma$ at some temperature, it is possible to estimate $E_{A}$ from Eq. (1) considering $\sigma_{0}=50 \mathrm{~S} / \mathrm{cm}$, and achieve a rough sketch of $\sigma$ at different temperatures. Or, if $E_{A}$ is obtained by some experimental or theoretical technique, ionic conductivity can be calculated.

Another "universal" curve, following Eq. (7) and considering some binary alkali silicate glasses, resulted in the same "universal" behavior [34], as cited above. The pre exponential value was $\sigma_{0}=50000 \mathrm{~K} \cdot \mathrm{S} / \mathrm{cm}$, considering the same conductivity data of Figs. $4 a-b$. The conclusions for this case also follows the above described considering Eq. (1). The most important fact is that in Figs. $4 a-b$ the scattered data by glasses of different compositions is unified by the single "universal" modified Arrhenius Eq. (1). The fact that $\sigma$ lies on this single "universal" curve for many ion-conducting glasses means that $\sigma$ is governed mainly by $E_{A}$.

The relations expressed in Eqs. (1) and (7) have an important note: no matter is the ionic conductor type, nor the oxide glass former, if one takes its conductivity $\log \sigma$ or $\log \sigma T$ 

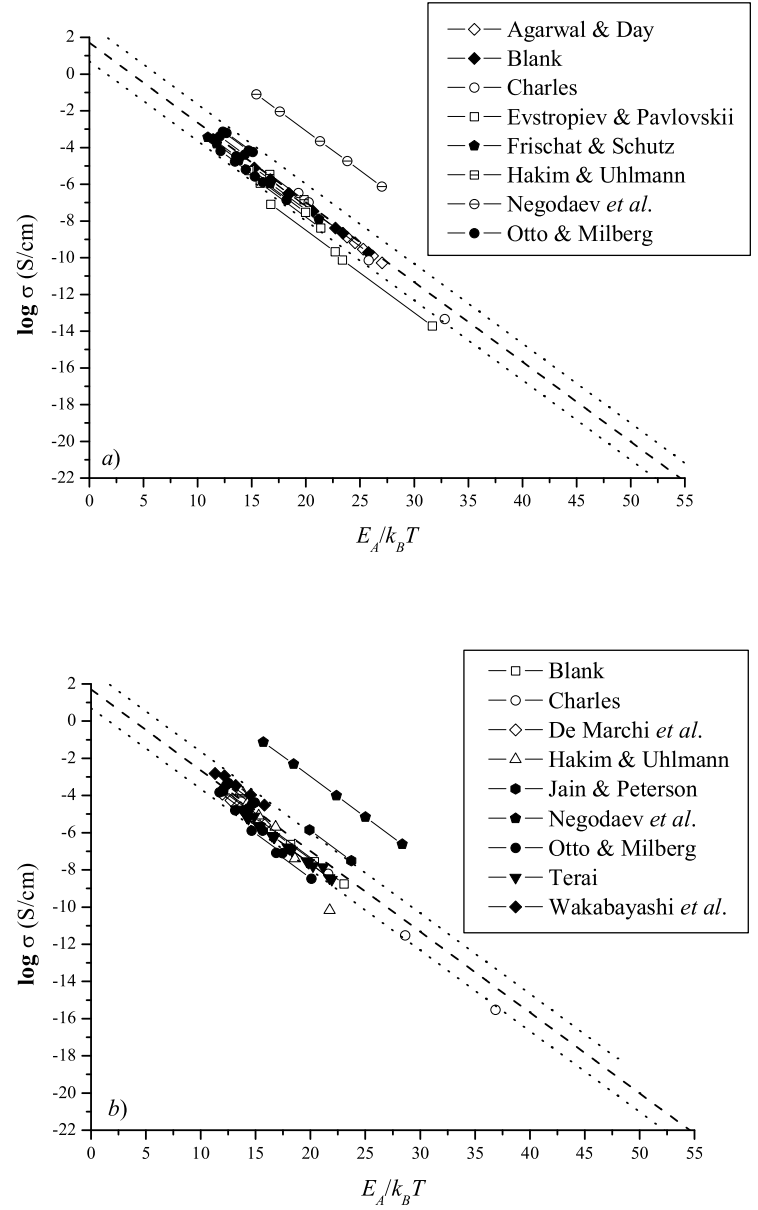

FIG. 4: Modified Arrhenius plots of ionic conductivities in 23 binary rubidium $(a)$ and 30 cesium $(b)$ silicate glasses. The dashed line is the "universal" curve, Eq. (1), with $\sigma_{0}=50 \mathrm{~S} / \mathrm{cm}$, and the dotted lines correspond to one order of magnitude higher or lower than Eq. (1).

and plots against $E_{A} / k_{B} T$, all systems will follow the same rule. In summary, there are strong connections between mi- croscopic structure and the ionic conductivity, fact based in the different types of composition in the alkali silicate glasses presented. Both alkali system compositions fall into an identifiable pattern where conductivity is related to structure, in the form expressed by the "volume pathways" of Adams \$ Swenson [35]. Further studies on other binary alkali oxide glasses as presented on Figs. $4 a-b$ would refuse or recognize this "universal" finding, to gain further insight into the nature of ion dynamics in glass.

\section{CONCLUSIONS}

The Anderson-Stuart model (A-S) has been used for rubidium and cesium -silica glass systems to describe the variation of the activation energy against composition in a wide composition range (from 5 up to $45 \mathrm{Rb}_{2} \mathrm{O}$ and $\mathrm{Cs}_{2} \mathrm{O}$ mole \%), with reasonable results. Theoretical activation energy $E_{A}$ of alkali silicate glasses seems to vary smoothly with composition. For the first time the considerations on experimental shear modulus $G$ and relative dielectric permittivity $\varepsilon$ with alkali composition have presented good fits on experimental available data. Relative dielectric permittivity $\varepsilon$ has giving more influence on fitting than $G$ in the alkali silicate glass systems studied. An "universal" finding is obtained using $\log \sigma \times E_{A} / k_{B} T$ in these binary silicate systems. At first sight $E_{A}$ and $k_{B} T$ are independent, as described by common Arrhenius plots, and $E_{A}$ varies strongly with composition (the effect of glass composition is demonstrated clearly by Figs. $3 a-b$ ). But modified Arrhenius plots showed that $E_{A} / k_{B} T$ is temperature and composition dependent and also related to $\sigma$. In other words, both $E_{A}$ and $k_{B} T$ (in the form $E_{A} / k_{B} T$ ) are related to the "volume pathway" factor $F$ of Adams and Swenson (Eq. (7)). Furthermore, frequency distribution values of pre exponential $\sigma_{0}$ showed an average result of $50 \mathrm{~S} / \mathrm{cm}$.

\section{Acknowledgments}

The authors acknowledge Brazilian agencies FAPESP, CAPES and CNPq for grant.
[1] C. A. Angell, Ann. Rev. Phys. Chem. 43, 693 (1992).

[2] D. Ravaine and J. L. Souquet, Phys. Chem. Glasses 18, 27 (1977).

[3] K. Funke, Prog. Solid State Chem. 22, 111 (1993).

[4] O. L. Anderson and D. A. Stuart, J. Am. Ceram. Soc. 37, 573 (1954).

[5] A. Bunde, M. D. Ingram, and P. Maass, J. Non-Cryst. Solids 172/174, 1222 (1994).

[6] R. M. Hakim and D. R. Uhlmann. Phys. Chem. Glasses 12, 132 (1971).

[7] D. K. McElfresh and D. G. Howitt, J. Am. Ceram. Soc. 69, C237 (1986)

[8] S. V. Nemilov, Zh. Fiz. Khim. 47, 1479 (1973).
[9] K. Matusita and S. Sakka, Proc. Xth Intern. Congr. on Glass, Kyoto (1974) 44

[10] J. E. Shelby and D. E. Day, J. Am. Ceram. Soc. 53, 182 (1970).

[11] K. Takahashi and A. Osaka, J. Ceram. Soc. Jpn. 91, 116 (1983).

[12] E. M. Amrhein, Glastech. Ber. 36, 425 (1963).

[13] R. M. Hakim and D. R. Uhlmann, Phys. Chem. Glasses 14, 81 (1973).

[14] K. Matusita, S. Sakka, A. Osaka, N. Soga, and M. Kunugi, J. Non-Cryst. Solids 16, 308 (1974).

[15] R. Terai, J. Non-Cryst. Solids 6, 121 (1971).

[16] R. J. Charles, J. Am. Ceram. Soc. 49, 55 (1966).

[17] A. K. Agarwal and D. E. Day, J. Am. Ceram. Soc. 65, 111 (1982). 
[18] K. Blank, Glastech. Ber. 39, 489 (1966).

[19] G. De Marchi, P. Mazzoldi, and A. Miotello, Proc. XVth Intern. Congr. on Glass, Leningrad (1989) 231.

[20] K. K. Evstropiev and V. K. Pavlovskii, Neorg. Mater. 3, 673 (1967).

[21] G. H. Frishat and H. -E. Schutz, Glastech. Ber. 49, 162 (1976).

[22] R. M. Hakim and D. R. Uhlmann, Phys. Chem. Glasses 8, 174 (1967).

[23] H. Jain and N. L. Peterson, J. Am. Ceram. Soc. 66, 174 (1983).

[24] G. D. Negodaev, I. A. Ivanovand, and K. K. Evstropiev, Neorg. Mater. 8, 342 (1972)

[25] K. Otto and M. E. Milberg, J. Am. Ceram. Soc. 51, 326 (1968).

[26] R. Terai, J. Ceram. Soc. Jpn. 77, 318 (1969).

[27] H. Wakabayashi, R. Terai, and H. Watanabe, J. Ceram. Soc.
Jpn. 94, 677 (1986).

[28] A. Doi, J. Mater. Sci. 39, 6827 (2004).

[29] J. Swenson and L. Börjesson, Phys. Rev. Lett. 77, 3569 (1996).

[30] S. Adams and J. Swenson, Phys. Chem. Chem. Phys. 4, 3179 (2002).

[31] J. Swenson and S. Adams, Phys. Rev. B64, 024204 (2002).

[32] S. Adams and J. Swenson, Solid State Ion. 154/155, 151 (2002).

[33] M. L. F. Nascimento and S. Watanabe, J. Mater. Sci. (2005) accepted.

[34] M. L. F. Nascimento and S. Watanabe, J. Mater. Sci. (2005) accepted.

[35] S. Adams and J. Swenson, Phys. Rev. Lett. 84, 4144 (2000). 